\title{
Spatial Analysis of People Living with HIV/AIDS Transmitted Through Commercial Heterosexual Contact or Non-Marital Non-Commercial Heterosexual Contact — China, 2018
}

\author{
Zhilong Dong ${ }^{1, x ;}$ Xiaohong Pan ${ }^{2,8}$; Chang Cai ${ }^{3}$; Qianqian Qin ${ }^{3}$; George F. Gao ${ }^{1, *,}$; Fan Lyu ${ }^{3, *}$
}

\section{Summary}

What is already known about this topic?

Significant changes in human immunodeficiency virus (HIV) transmission modes have occurred in China, and the proportion of heterosexual transmission increased in recent years.

What is added by this report?

The proportions of diverse transmission routes and subgroups of heterosexual transmission were analyzed by provincial-level administrative divisions (PLADs), and nationwide spatial clustering of HIV transmission through commercial heterosexual contact $(\mathrm{CHC})$ and non-marital non-commercial heterosexual contact (NMNCHC) was explored.

What are the implications for public health practice?

This report provides evidence for geographic clustering of HIV transmission through CHC and NMNCHC in China and identifies priority regions where specified research and targeted HIV prevention and control strategies should be implemented.

Over recent years, significant changes in human immunodeficiency virus (HIV) transmission modes have occurred in China, and the proportion of sexually transmitted infections has increased greatly (1). In addition, both the number of reported cases of people living with HIV/AIDS (PLWHA) and the distribution of diverse HIV transmission routes display regional characteristics (2). However, the nationwide distribution of heterosexual transmission subtypes remains unclear, including commercial heterosexual transmission, non-marital non-commercial heterosexual transmission, and marital heterosexual transmission (3).

Fully understanding the spatial characteristics of heterosexual transmission could establish a basis to formulate targeted regional prevention and control measures. For this purpose, this study used data from the Chinese HIV/AIDS Comprehensive Response Information Management System (CRIMS) to analyze the spatial distribution characteristics of the two main modes of heterosexual transmission, including transmission through commercial heterosexual contact $(\mathrm{CHC})$ and non-marital non-commercial heterosexual contact (NMNCHC).

Newly identified cases of HIV infection were reported through the web-based CRIMS by individuals from local CDCs and medical institutions (4). Data usage from CRIMS was authorized by the National Center for AIDS/STD Control and Prevention of China CDC. All newly identified PLWHA in 2018 in the CRIMS were included in our study.

The frequency distribution and the constituent ratio of PLWHA infected through various transmission routes and the subgroups of those infected through heterosexual transmission were analyzed in each provincial-level administrative divisions (PLADs). Moreover, Moran's I index was utilized to explore the clustering patterns of PLWHA identified in 2018 in China, with the assumption that cities throughout the country were not significantly different from each other with respect to the spatial distribution of infection. Subsequently, Z-scores with corresponding $P$-values were presented. Statistically significant Moran's I indexes revealed the presence of overall nationwide clustering. After a national level of clustering was confirmed, local clustering analysis was required to detect specific clustering areas. Hotspot analysis (Getis-Ord Gi*) in ArcGIS (version 13.0, Esri Inc, Redlands, CA, USA) was conducted to determine areas with significantly high clusters and areas with significantly low clusters, using 3 levels of confidence intervals (5).

In this study, PLADs with large numbers of newly identified PLWHA normally have high proportions of heterosexual transmission. In 2018, heterosexual transmission accounted for $71.5 \%$ of all transmission. Among the top 10 PLADs with the highest numbers of 
reported PLWHA, the proportions of heterosexual transmission were higher than average in 7 PLADs, including Guizhou (92.5\%), Guangxi (91.2\%), Yunnan (89.8\%), Xinjiang (85.6\%), Chongqing (83.2\%), Sichuan (83.0\%), and Hunan (78.6\%), while those of Henan (67.3\%), Guangdong (62.4\%), and Zhejiang $(58.9 \%)$ were below average. In addition to these top 10 PLADs, the proportions of heterosexual transmission in most other PLADs were below average, except for Xinjiang Production and Construction Corps (XPCC) $(87.8 \%)$ and Jiangxi $(84.4 \%)$, where the numbers of cases were relatively smaller than those of other regions (Figure 1A, Figure 1B).

In general, the proportion of newly identified PLWHA transmitted through NMNCHC (49.0\%) was higher than through $\mathrm{CHC}(38.0 \%)$ in heterosexual transmission, and the proportion of $\mathrm{NMNCHC}$ related transmission was higher than that of $\mathrm{CHC}$ in most PLADs and regions in 2018. Among the top 10 PLADs with the highest number of reported PLWHA, the ratio of NMNCHC to $\mathrm{CHC}$ transmission was greater than 1 in 7 PLADs, with Xinjiang having the highest ratio $(5.94: 1)$, followed by other PLADs such as Guizhou (2.74:1), Yunnan (2.19:1), Henan $(1.27: 1)$, Guangdong $(1.18: 1)$, Sichuan $(1.05: 1)$, and Hunan $(1.03: 1)$. Those with a ratio less than 1 included Zhejiang $(0.9: 1)$, Chongqing $(0.87: 1)$, and Guangxi (0.56:1) (Figure 1C, Figure 1D).

General spatial autocorrelation was conducted for newly identified PLWHA transmitted through CHC and NMNCHC in China in 2018. Moran's I indexes were greater than 0 and the $\mathrm{Z}$-values were greater than 1.96 for both transmission routes $(P<0.0001)$. These results confirmed the potential existence of nationwide HIV/AIDS epidemic clustering for PLWHA transmitted through CHC and NMNCHC (Table 1).

In 2018, 373 cities reported PLWHA transmitted through heterosexual contact, among which cases transmitted through NMNCHC were reported in 371 cities, while PLWHA transmitted through CHC were reported in 360 cities. Furthermore, 13 cities had identified more than 500 cases of PLWHA reported as transmitted via $\mathrm{CHC}$, located in Chongqing, Sichuan, Guangxi, and Yunnan. In contrast, 20 cities had more than 500 PLWHA reported as being transmitted through NMNCHC, including Chongqing, Sichuan, Yunnan, Xinjiang, Guizhou, Guangdong, and Beijing.

The Getis-Ord Gi* statistics revealed that at the 95\% confidence level, hotspots of PLWHA infected through $\mathrm{CHC}$ were mainly discovered in Sichuan, Chongqing, Guizhou, Guangxi, northeast Yunnan, west Guangdong, west Hunan, southwest Hubei, southeast Gansu, and southwest Shaanxi, whereas coldspots were observed in large areas of northeastern and northern China, comprised of more than 10 PLADs such as Shandong, Hebei, Tianjin, and Beijing. Hotspots for PLWHA infected through NMNCHC were detected mainly in Sichuan, Chongqing, Yunnan, Guizhou, and the western regions of Guangxi, while cold-spots were observed in eastern Hebei, Tianjin, Shandong, Anhui, northern Jiangsu, eastern Henan, and a small part of western Jilin.

\section{DISCUSSION}

This analysis demonstrated that the overall prevalence of HIV in China follows inconsistent distribution patterns across different PLADs. In general, PLADs with higher annual frequencies of newly reported PLWHA have higher proportions of heterosexual transmission. Additionally, the distribution of PLADs and cities is unbalanced in the subclassification of heterosexual transmission. In some regions, transmission via $\mathrm{CHC}$ is higher than NMNCHC, while in others, the proportion of transmission through $\mathrm{NMNCHC}$ is higher. These findings were consistent with the results of other regional studies $(6-7)$.

There was a certain degree of overlap among cities with higher numbers of reported cases transmitted through $\mathrm{CHC}$ and $\mathrm{NMNCHC}$, but $\mathrm{CHC}$ and NMNCHC also displayed unique characteristics with respect to the distribution of hotspots. The distribution of NMNCHC was relatively more concentrated, while that of $\mathrm{CHC}$ exhibited a wider pattern of distribution. This could be explained by the fact that $\mathrm{NMNCHC}$ was more sporadic than $\mathrm{CHC}$ and hence more likely to cluster within areas where there was high HIV prevalence. In addition, $\mathrm{CHC}$ was more frequent in eastern China, covering some parts of Guangxi and Guangdong, while NMNCHC was more prevalent in western China, mainly concentrated in the southwest. This may indicate that heterosexual commercial services were comparatively more available in some areas of Guangdong or Guangxi. Further research is essential to determine the underlying causes of CHC hotspots in these areas to develop targeted prevention and control strategies.

The hotspots of $\mathrm{CHC}$ and NMNCHC also overlapped to a large extent, and approximately $80 \%$ of the hotspots of NMNCHC were overlapped with those of CHC. There was likely some interweaving 


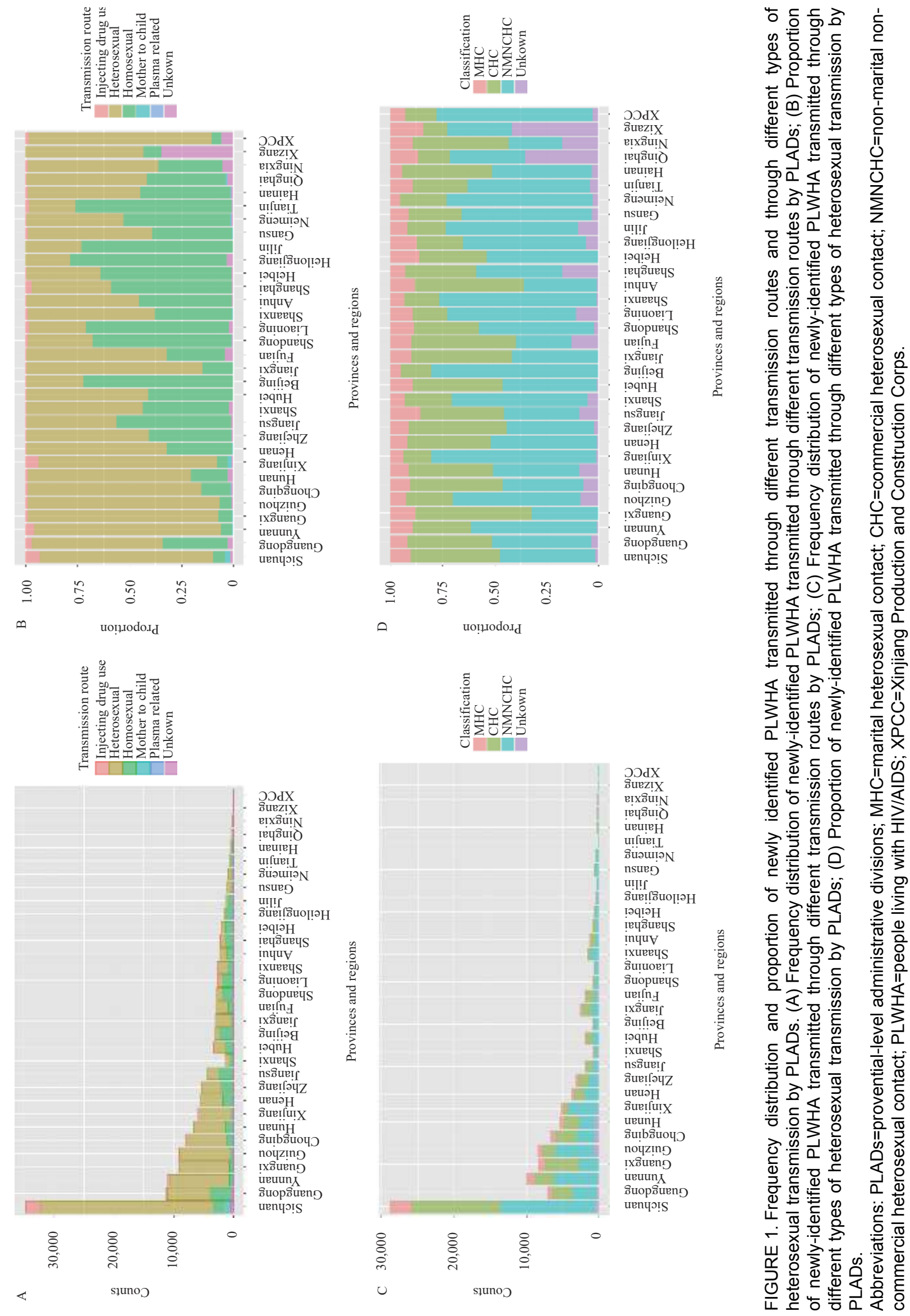


TABLE 1. Results of general spatial autocorrelation for the newly identified PLWHA reported as transmitted through $\mathrm{CHC}$ and NMNCHC in 2018 in China.

\begin{tabular}{cccc}
\hline Transmission routes & Moran's I & Z-value & $\boldsymbol{P}$-value \\
\hline $\mathrm{CHC}$ & 0.2916 & 22.33 & $<0.0001$ \\
$\mathrm{NMNCHC}$ & 0.1847 & 15.32 & $<0.0001$
\end{tabular}

Abbreviations: $\mathrm{CHC}=$ commercial heterosexual contact; $\mathrm{NMNCHC}=$ non-marital non-commercial heterosexual contact; PLWHA=people living with HIVIAIDS.

between these two populations. For example, some men might be engaged in both commercial and nonmarital non-commercial heterosexual behaviors, which may transmit HIV from infected female sex workers (FSWs) to uninfected women in the general population, thereby spreading HIV from high-risk groups to the general population through heterosexual transmission (8).

However, in the coastal areas of China, no hotspot for $\mathrm{CHC}$ or NMNCHC was identified. This may be because the frequency of HIV transmission in coastal areas was relatively low, and the distribution density of the HIV epidemic was relatively small. Consequently, there were fewer sexually transmitted diseases. Nonetheless, this could also be because some large cities in areas such as Beijing or Tianjin have a high prevalence of men who have sex with men (MSM) transmission, accounting for the majority of newly identified PLWHA (9-10). Furthermore, this study discovered that although the overall proportion of heterosexual transmission in Beijing or Tianjin was comparatively small, NMNCHC remained the principal mode of transmission via heterosexual contact in these areas. Further research is necessary to evaluate the relationship between MSM groups and those cases reported as infected through $\mathrm{CHC}$ or NMNCHC.

This study was subject to some limitations. First, the history of sexual contact in the CRIMS is self-reported, which could lead to inaccurate reports due to social desirability, stigmatization, or recall bias. Second, different regions have inconsistent rates of identified cases, which may influence conclusions. However, a large sample size and nationwide coverage could compensate for these deficiencies to some extent.

In summary, this study provided evidence for the geographic clustering of HIV transmission through $\mathrm{CHC}$ and $\mathrm{NMNCHC}$ in China and identifies priority regions where specified research and targeted HIV prevention and control measures should be implemented. doi: $10.46234 / \mathrm{ccdcw} 2021.086$

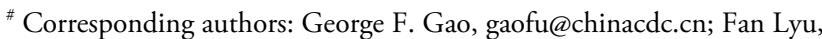
fanlv@chinaaids.cn.

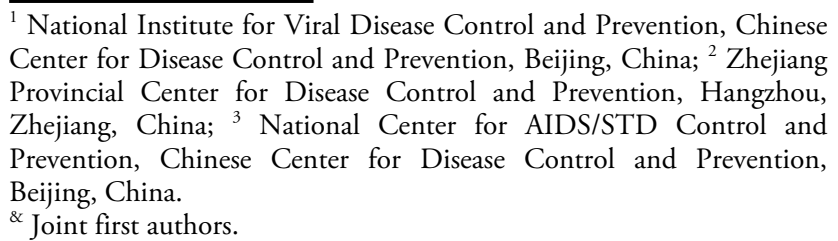

Submitted: January 24, 2021; Accepted: April 02, 2021

\section{REFERENCES}

1. Wu ZY. Characteristics of HIV sexually transmission and challenges for controlling the epidemic in China. Chin J Epidemiol 2018;39(6):707 9. http://dx.doi.org/10.3760/cma.j.issn.0254-6450.2018.06.002. (In Chinese).

2. Qian SS, Guo W, Xing JN, Qin QQ, Ding ZW, Chen FF, et al. Diversity of HIV/AIDS epidemic in China: a result from hierarchical clustering analysis and spatial autocorrelation analysis. AIDS 2014;28(12):1805 - 13. http://dx.doi.org/10.1097/qad.00000000000 00323.

3. Dong ZL, Ma LY, Cai C, Gao GF, Lyu F. Demographic features of identified PLWHA infected through commercial and nonmarital noncommercial heterosexual contact in China from 2015 to 2018: a retrospective cross-sectional study. BMC Infect Dis 2021;21(1):71. http://dx.doi.org/10.1186/s12879-020-05757-2.

4. Zhang XY, Huang T, Feng YB, Li M, Chen FF, Li YG, et al. Characteristics of the HIV/AIDS epidemic in women aged 15-49 years from 2005 to 2012 in China. Biomed Environ Sci 2015;28(10):701 8. http://dx.doi.org/10.3967/bes2015.100.

5. Okoli ML, Alao S, Ojukwu S, Emechebe NC, Ikhuoria A, Kip KE. Predictive and spatial analysis for estimating the impact of sociodemographic factors on contraceptive use among women living with HIV/AIDS (WLWHA) in Kenya: implications for policies and practice. BMJ Open 2019;9(1):e022221. http://dx.doi.org/10.1136/ bmjopen-2018-022221.

6. Yu QY, Xu P, Lin P, Li Y, Wang LH, Li DD, et al. Epidemiological characteristics and latent class analysis of non-marital heterosexual behaviors among human immunodeficiency virus/acquired immunodeficiency syndrome individuals in Jiangmen, Guangdong Province between 2015 and 2017. Chin J Prev Med 2018;52(12): 1269 - 75. http://dx.doi.org/10.3760/cma.j.issn.0253-9624.2018.12. 015. (In Chinese).

7. Yu QY, Wang FL, Xu P, Wen HJ, Xiong YX, Yang J, et al. Characteristics of non-marital and non-commercial heterosexual transmission of HIV infection in Miao-Dong Autonomous prefecture of Qiandongnan. Chin J Prev Med 2017;51(11):977 - 81. http://dx. doi.org/10.3760/cma.j.issn.0253-9624.2017.11.005. (In Chinese).

8. Wu PL, Dong WM, Rou KM, Dong W, Zhou C, Chen X, et al. HIVpositive clients of female sex workers in Hunan Province, China: a mixed methods study assessing sexual relationships and risk behavior by type of partner. BMC Public Health 2019;19(1):1129. http://dx. doi.org/10.1186/s12889-019-7446-1.

9. Zheng MN, Yu MH, Cheng SH, Zhou N, Ning TL, Li L, et al. Characteristics of HIV-1 molecular transmission networks and drug resistance among men who have sex with men in Tianjin, China (20142018). Virol J 2020;17(1):169. http://dx.doi.org/10.1186/s12985-02001441-8.

10. Wang J, He SF, Li Y, Lu HY. Analysis of epidemiological characteristics of HIV/AIDS in Beijing, 2017. Cap J Public Health 2018;12(6):282 4. http://dx.doi.org/10.16760/j.cnki.sdggws.2018.06.002. (In Chinese). 